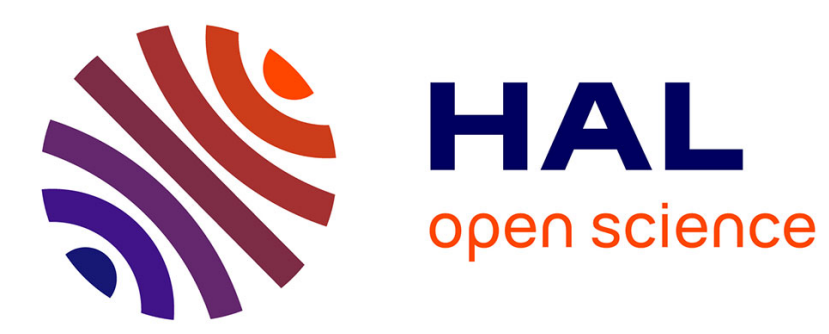

\title{
Experimental results on MMSE turbo equalization in underwater acoustic communication using high order modulation
}

Christophe Laot, Nicolas Beuzeulin, Arnaud Bourre

\section{To cite this version:}

Christophe Laot, Nicolas Beuzeulin, Arnaud Bourre. Experimental results on MMSE turbo equalization in underwater acoustic communication using high order modulation. Oceans'10, Sep 2010, Seattle, United States. hal-00523104

\author{
HAL Id: hal-00523104 \\ https://hal.science/hal-00523104
}

Submitted on 10 Jun 2021

HAL is a multi-disciplinary open access archive for the deposit and dissemination of scientific research documents, whether they are published or not. The documents may come from teaching and research institutions in France or abroad, or from public or private research centers.
L'archive ouverte pluridisciplinaire $\mathbf{H A L}$, est destinée au dépôt et à la diffusion de documents scientifiques de niveau recherche, publiés ou non, émanant des établissements d'enseignement et de recherche français ou étrangers, des laboratoires publics ou privés. 


\title{
Experimental results on MMSE turbo equalization in underwater acoustic communication using high order modulation
}

\author{
Christophe Laot*, Nicolas Beuzelin ${ }^{\dagger}$ and Arnaud Bourré ${ }^{\dagger}$ \\ * Institut TELECOM ; TELECOM Bretagne ; LabSTICC CNRS UMR 3192, \\ Université européenne de Bretagne, \\ Technopole Brest-Iroise - CS 83818 - 29238 Brest Cedex 3, France \\ Email: christophe.laot@telecom-bretagne.eu \\ ${ }^{\dagger}$ DGA/GESMA, BCRM Brest, CC 42, 29240 Brest Cedex, France \\ Email: nicolas.beuzelin@dga.defense.gouv.fr, arnaud.bourre@dga.defense.gouv.fr
}

\begin{abstract}
This paper presents some results on MMSE turbo equalization applied to real sea water trials in the Atlantic ocean. High order modulations (8-PSK, 16-QAM and 32-QAM) have been tested. The channel data rate on the underwater acoustic channel is up to $20 \mathrm{kbps}$. The receiver includes timing recovery, equalization, interleaving and channel coding. The results which are evaluated in term of bit error rate (BER) and mean square error (MSE) show that turbo equalization improves the performance of the receiver and allows reliable high order transmissions when the signal to noise ratio is sufficient.
\end{abstract}

\section{INTRODUCTION}

Wireless information transmissions through the ocean need reliable underwater acoustic communications. One of the numerous applications of the underwater communication is to provide a high data rate link between an autonomous underwater vehicle (AUV) and a surface vessel. GESMA (Groupe d'Etudes Sous-Marines de l'Atlantique, Brest, France), in collaboration with Telecom Bretagne and SERCEL, developed an acoustic link, called TRIDENT [1]. This system was designed for text, images and speech data transmission in a shallow water environment.

The technical specifications of the TRIDENT system are the following. In order to maximize the spectral efficiency of the transmission, a single carrier scheme and a continuous transmission model was designed. This scheme avoids the spectral efficiency loss due to the insertion of guard interval or cyclic prefix as required in a classical OFDM scheme. In order to minimize the use of training sequences, blind or decision directed algorithms are used to track the time-varying channel.

High data rate communication over a distance greater than one $\mathrm{km}$ induces a low signal to noise ratio (SNR) at the receiver side. Multiple antennas were considered at the receiver side and multiple input equalization was designed to improve the receiver performance. An efficient timing recovery scheme was developed to compensate for the Doppler effect induced by the moving platforms. Channel coding was integrated in

This work was supported in part by GESMA/DGA, France. the transmission scheme to increase the robustness of the transmission. Nevertheless, when the received SNR is too low, the classical transmission scheme fails and the transmission is unreliable.

Minimum mean square error (MMSE) turbo equalization [2]-[4] has proven to be effective for removing intersymbol interference and improves the performance of the receiver in low SNR situations. It consists of a MMSE equalizer and a decoder which exchange soft information in an iterative process. In a turbo equalizer, the equalizer benefits from the channel decoder gain thanks to an iterative process applied to the same data block. The MMSE turbo equalization has already been proposed in UWA communications [5]-[8]. The sections I-III are similar to those of the previous paper [9]. The contribution of this paper essentially appears in the section IV where new results are given on the performance of the turbo equalization using high order modulations (8-PSK, 16-QAM and 32-QAM).

\section{SYSTEM MODEL}

This paper proposes a multiple input adaptive MMSE turbo equalization scheme for an all-digital single carrier receiver in a time-varying channel environment. The receiver has a chain of $N_{R}$ antennas.

A rate $R_{c}$ convolutional code is fed by a block of binary data $\alpha_{n}$. An interleaver $\Pi$ shuffles a block of coded data $c_{n, i}$ and provides an interleaved coded data $c_{k, i}$ block. Each set of $m=\log _{2}(M)$ interleaved coded data $c_{k, i}$ is associated with M-ary complex symbol $d_{k}$ by a Gray mapping. Long burst transmission results on the concatenation of one preamble followed by a repetition of blocks of symbols separated by a pilot sequence.

Let $s(t)$ be the transmitted waveform of the QPSK signal:

$$
s(t)=\Re e\left\{\sum_{n=-\infty}^{+\infty} d_{k} g(t-k T) e^{j\left(2 \pi f_{c} t+\psi\right)}\right\}
$$




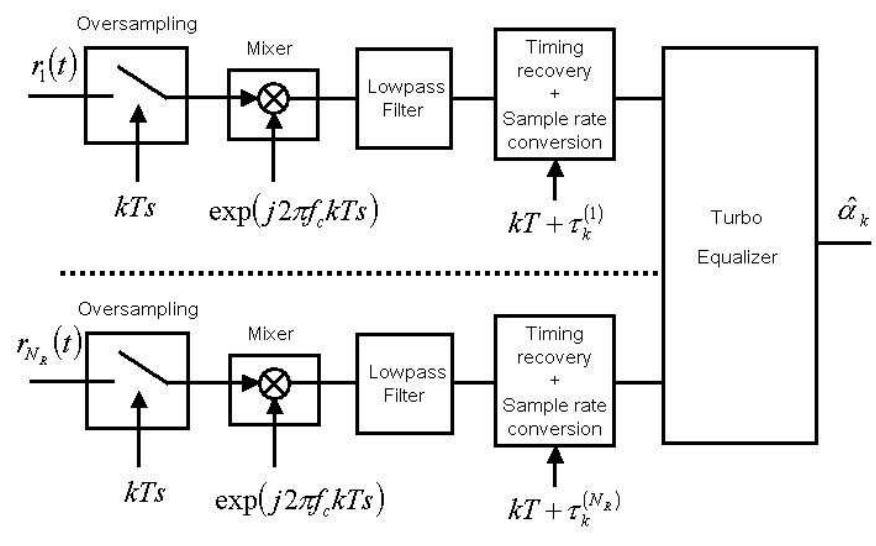

Fig. 1. All-digital receiver

where $f_{c}$ is the carrier frequency, $\psi$ is the carrier phase uncertainty, $1 / T$ is the symbol rate with $T$ the symbol duration, $\left\{d_{k}\right\}$ are the transmitted symbols with variance $\sigma_{d}^{2}$ and $g(t)$ is a square root raised cosine filter with roll-off factor 0.25 .

When the pass-band digital received signal is centred on a relatively low carrier frequency, an all-digital receiver can be performed [10]. Since the received signal is band-limited, an oversampling is performed at the rate $1 / T_{s}$ where $T_{s}$ is chosen to respect the sampling theorem. The down-conversion is performed digitally and the optimum sampling is realized from a sample rate converter. The resulting all-digital receiver is depicted in Figure 1.

In wide-band transmission, as it is the case in underwater acoustic communications [11], [12], the Doppler effect introduces a scaling of the symbol period and must be taken into account in the design of the timing recovery scheme [13], [14]. To restore the transmitted signal, the optimum sample time not only depends on the propagation delay at the antenna $j ; j=1, \ldots, N_{R}$ but also on a common Doppler shift function on the relative speed of the platforms and the propagation wave velocity [13]-[16]. Because the receiver is all-digital, the optimum sampling value $k T+\tau_{k}^{(j)}$ does not necessary concur with a value of $k T_{s}$ and it will be needed to proceed to a sample rate conversion. The sample rate conversion uses interpolation, filtering and decimation [10].

At the receiver side, the optimum sampling time is unknown and must be estimated. Initial compensation of common Doppler shift is performed on the estimation on the relative velocity measured using a short preamble [17] transmitted at the beginning of the transmission. Note that the preamble is also used to realize the transmission detection and frame synchronization. Then, an NDA timing recovery tracking scheme is designed which takes into account the residual Doppler shift due to the moving platforms and the different channel delays on each antenna [15], [18], [19].

After demodulation, sampling, Doppler compensation, timing recovery, the received signal is modelled by the output of a single input multiple output (SIMO) discrete time channel where each output $j ; j=1, \ldots, N_{R}$ is corrupted by an additive, zero-mean, white Gaussian noise (AWGN) $w_{k}^{(j)}$ with variance

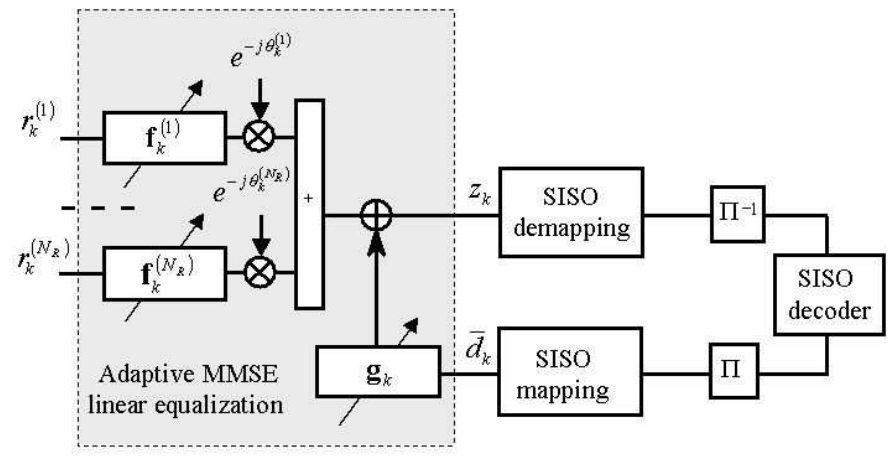

Fig. 2. Turbo-equalizer

$\sigma_{(j)}^{2}$. The receiver antenna $j$ observes the input $r_{k}^{(j)}$

$$
r_{k}^{(j)}=\sum_{l=0}^{L^{(j)}} h_{k, l}^{(j)} d_{k-l}+w_{k}^{(j)}
$$

where $h_{k, l}^{(j)}$ are the $L^{(j)}+1$ coefficients of the multi-path timevarying channel seen at the antenna $j$ at the time $k T$.

\section{TURBO-EQUALIZATION PRINCIPLE}

In turbo equalization, the equalization and the channel decoding are jointly performed in an iterative way. Each iteration $p$ is carried out by a module fed in by both received signal samples $r_{k}^{(j)}$ and estimated data $\bar{d}_{k}$ originating from the module p-1. As depicted in Figure 2, each module consists of an multiple-input equalizer, a soft input soft output (SISO) demapper, a deinterleaver $\Pi^{-1}$, a SISO binary decoder, an interleaver $\Pi$ and a SISO mapper. Each module $p$ delivers an estimate of the symbol $d_{k}$, called $\bar{d}_{k}$, to be used by the equalizer of the module $p+1$.

\section{A. SISO mapping}

Transmitted symbols are unknown at the receiver side, but it is possible to get an estimated soft value from log likelihood ratio (LLR) on the coded bits provided by the SISO channel decoder of the previous module. This paragraph proposes a solution to produce an M-ary symbol from the binary SISO decoder.

This operation involves calculating the soft estimate $\bar{d}_{k}$, defined as the mathematical expectation of symbol $d_{k}$ and given by $\bar{d}_{k}=\sum_{M} d \times P_{a}\left(d_{k}=d\right)$. The sum here concerns all of the $M$ discrete symbols $d$ in the constellation. The term $P_{a}\left(d_{k}=d\right)$ denotes the a priori probability and depends on probability of the encoded bits $c_{k, i} ; i=1, \ldots, m$ making up symbol $d_{k}$. When the encoded bits are statistically independent, it is possible to write:

$$
P_{a}\left(d_{k}\right)=\prod_{i=1}^{m} P_{a}\left(c_{k, i}\right)
$$

where binary element $c_{k, i}$ takes the value 0 or 1 according to the considered symbol $d_{k}$ and the mapping rule. On the other 
hand, it can be shown that the a priori probability and the $a$ priori LLR are linked by the following relation:

$$
P_{a}\left(c_{k, i}\right)=\frac{1}{2}\left(1+\left(2 c_{k, i}-1\right) \tanh \left(\frac{L_{a}\left(c_{k, i}\right)}{2}\right)\right)
$$

where $L_{a}\left(c_{k, i}\right)$ defines a priori information on the encoded bits $c_{k, i}$ coming from the channel decoder.

\section{B. Adaptive multiple-input equalizer structure}

The structure of the equalizer is depicted in Figure 2. The multiple-input equalizer combines the outputs of the feedforward transversal filters fed by the SIMO channel. Second order phase lock loops (PLL) are optimized jointly to the equalizer filters in order to compensate residual frequency offsets. When a priori information are available from the channel decoder of a previous iteration, intersymbol interference at the combiner output is suppressed by the output of a feedback filter fed in by the estimated symbols $\bar{d}_{k}$.

The filter coefficients of the equalizer are optimized so as to minimize the mean square error $E\left\{\left|z_{k}-d_{k-\Delta}\right|^{2}\right\}$ between the equalized symbol $z_{k}$ at instant $k$ and symbol $d_{k-\Delta}$ transmitted at instant $k-\Delta$. The introduction of a delay $\Delta$ enables the anti-causality of the solution to be taken into account.

An adaptive procedure is used to obtain the filter coefficients [2]. This adaptive algorithm is composed of two distinct phases: the training phase and the tracking phase. The training phase makes use of sequences known by the receiver (dataaided (DA)) to initialize the equalizer coefficients. Next, during the tracking period, the coefficients are continuously updated in a decision-directed (DD) manner, based on the receiver estimate of the transmitted sequence. Alternative to the DD algorithm makes use of blind algorithms when the signal to noise ratio is too low.

Adaptive algorithms involve determining, for each symbol entering the equalizer, output $z_{k}$ from the following relation:

$$
z_{k}=\left(\mathbf{f}_{k}^{(j)}\right)^{T} \mathbf{r}_{k}^{(j)} e^{-j \theta_{k}^{(j)}}-\mathbf{g}_{i}^{T} \tilde{\mathbf{d}}_{k}
$$

where $\tilde{\mathbf{d}}_{k}=\left(\bar{d}_{k+G}, \ldots, \bar{d}_{k-\Delta+1}, 0, \bar{d}_{k-\Delta-1}, \ldots, \bar{d}_{k-G}\right)^{T}$ is the vector of estimated symbols and $\mathbf{r}^{(j)} k_{k}=$ $\left(r_{k+F}^{(j)}, \ldots, r_{k-F}^{(j)}\right)^{T}$ is the vector of channel output samples, with respective lengths $2 F+1$ and $2 G+1$. Note that the coordinate relative to the soft estimate $\bar{d}_{k-\Delta}$ in $\tilde{\mathbf{d}}_{k}$ is set to zero in order not to cancel the signal of interest. Vectors $\mathbf{f}_{k}^{(j)}=\left(f_{k+F}^{(j)}, \ldots, f_{k-F}^{(j)}\right)^{T}$ and $\mathbf{g}_{k}=\left(g_{k+G}, \ldots, g_{k-G}\right)^{T}$ represent the coefficients of the filters $\mathbf{f}^{(j)}$ and $\mathbf{g}$, respectively. Both vectors are a function of time since they are updated at each new received symbol.

The relations used to update the vectors of the coefficients can be obtained from a least-mean square (LMS-DA) gradient algorithm:

$$
\begin{aligned}
\mathbf{f}_{k+1}^{(j)} & =\mathbf{f}_{k}^{(j)}-\mu\left(z_{k}-d_{k-\Delta}\right)\left(\mathbf{r}_{k}^{(j)} e^{-j \theta_{k}^{(j)}}\right)^{*} \\
\mathbf{g}_{k+1} & =\mathbf{g}_{k}+\mu\left(z_{k}-d_{k-\Delta}\right) \tilde{\mathbf{d}}_{k}^{*}
\end{aligned}
$$

where $\mu$ is a small, positive, step-size that controls the convergence properties of the algorithm.
During the tracking period, the LMS-DA algorithm is replaced by the LMS-DD algorithm where $d_{k-\Delta}$ is replaced by its estimate $\hat{d}_{k-\Delta}$ computed by decision on the equalizer output $z_{k}$.

During the first iteration of the turbo equalizer, $\tilde{\mathbf{d}}_{k}$ is a null vector. The result is that the vector $\mathbf{g}_{k}$ is also null. The MMSE equalizer then converges adaptively towards an MMSE transversal equalizer. When the estimated data are very reliable and close to the transmitted data, the MMSE equalizer converges towards an ideal (genie) interference canceller, then having the performance of a transmission without intersymbol interference [20].

We have therefore defined an adaptive MMSE turbo equalizer whose coefficients are obtained from a low complexity stochastic gradient descent algorithm, making it possible to track the slow time variations of the transmission channel.

\section{SISO demapping}

The role of this module is to convert the equalized data $z_{k}$ into extrinsic LLRs on the interleaved coded bits, which will be then transmitted to the channel decoder. Generally, we can always decompose the expression of $z_{k}$ as the sum of two quantities:

$$
z_{k}=g_{0} d_{k-\Delta}+\nu_{k}
$$

The term $g_{0} d_{k-\Delta}$ represents the useful signal up to a constant factor $g_{0}$. We recall that this factor quite simply corresponds to the central coefficient of the cascading of the channel with the equalizer. The term $\nu_{k}$ accounts for both residual interference and noise at the output of the equalizer. Using a Gaussian approximation of residual ISI, $\nu_{k}$ follows a complex Gaussian distribution, with zero mean and total variance bounded by $\sigma_{\nu}^{2}=\sigma_{d}^{2} g_{0}\left(1-g_{0}\right)$.

When Gray mapping rules are used, the extrinsic information on the coded bits is given by:

$$
L_{e}\left(c_{k-\Delta, i}\right)=\ln \frac{\sum_{d_{k-\Delta} \mid c_{k-\Delta, i}=1} \exp \left(-\frac{\left|z_{k}-g_{0} d_{k-\Delta}\right|^{2}}{\sigma_{\nu}^{2}}\right)}{\sum_{d_{k-\Delta} \mid c_{k-\Delta, i}=0} \exp \left(-\frac{\left|z_{k}-g_{0} d_{k-\Delta}\right|^{2}}{\sigma_{\nu}^{2}}\right)}
$$

To compute the extrinsic information $L_{e}\left(c_{k-\Delta, i}\right)$, we need bias $g_{0}$. From (7) and the expression of $\sigma_{\nu}^{2}$, it can be easily shown that $g_{0} \sigma_{d}^{2}=E\left\{\left|z_{k}\right|^{2}\right\}$. So, the factor $g_{0}$ is estimated by the mean square modulus of the equalizer output which is update for each block of symbols.

\section{SISO channel decoder}

The channel decoder is a SISO (Soft Input Soft Output) device which implements the LOG-MAP algorithm. The observations provided by the SISO demapper fed the channel decoder input which delivers soft output decisions on coded data. This soft output decisions fed the SISO mapper in order to provide estimated symbols. 


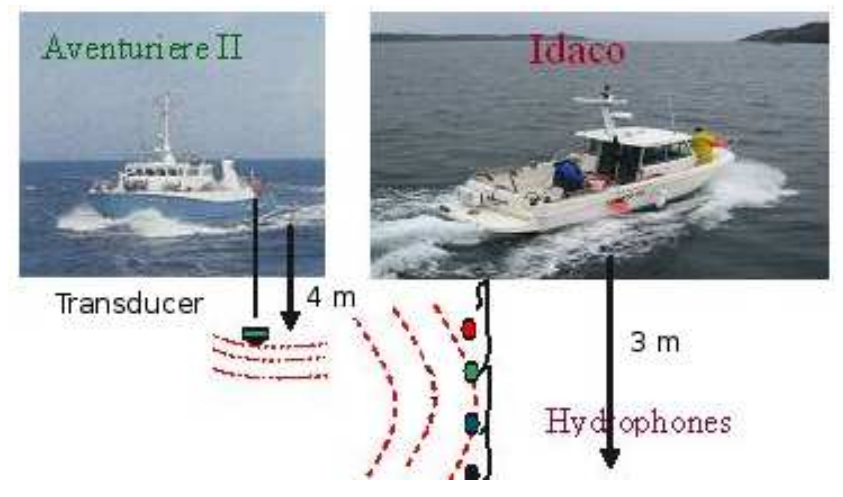

Fig. 3. Sea trial configuration

\section{SIMULATION AND EXPERIMENTAL RESULTS}

Our simulations emphasize the performance of the MMSE turbo equalizer over real sea trials. Experimental sea trials were carried out on March 2010 in the site "bay of Brest", France, by DGA/GESMA. The aim of these sea trials was to transmit data from a ship to another boat in a shallow water environment with a water depth of 10 to 30 meters. These trials were carried out in a context of rough sea. As depicted in the figure 3, the transmitter was placed on the board of the "Aventurière II" and the receiver on board of the boat "Idaco". At the receiver side, the antenna array was a vertical chain of $N_{R}=4$ hydrophones with a $25 \mathrm{~cm}$ distance.

During these trials, two particular sequences were recorded (AIT44 and AIT63) with the objective to test the turboequalization with high order modulations. Each recorded sequence included consecutive bursts of symbols (8PSK, 16QAM and 32QAM) separated by a guard interval of 5 seconds. Single carrier transmissions with a carrier frequency of $17.5 \mathrm{kHz}$ was used. The symbol rate was 4800 symbols per seconds. Figure 4 represents the position of the two ships during the transmission of the sequence named AIT63 (transmitter: green, receiver :red). The features of the two recorded sequences are given in Table I. Note that the relative velocity between the transmitter and the receiver was up to $v=2 \mathrm{~m} / \mathrm{s}$ for the sequence AIT63.

TABLE I

FEATURES OF THE REAL SEA TRIALS

\begin{tabular}{|l|c|c|}
\hline Sequence & AIT44 & AIT63 \\
\hline distance $(\mathbf{m})$ & 1530 & 640 \\
\hline $\mathbf{v}(\mathbf{m} / \mathbf{s})$ & 0.26 & 2.02 \\
\hline
\end{tabular}

TABLE II

FEATURES OF THE REAL SEA TRIALS

\begin{tabular}{|l|c|c|c|}
\hline Modulation type & 8-PSK & 16-QAM & 32-QAM \\
\hline Channel Bit Rate (bits/s) & 14400 & 19200 & 24000 \\
\hline User Bit Rate (bits/s) & 6480 & 8640 & 10800 \\
\hline
\end{tabular}

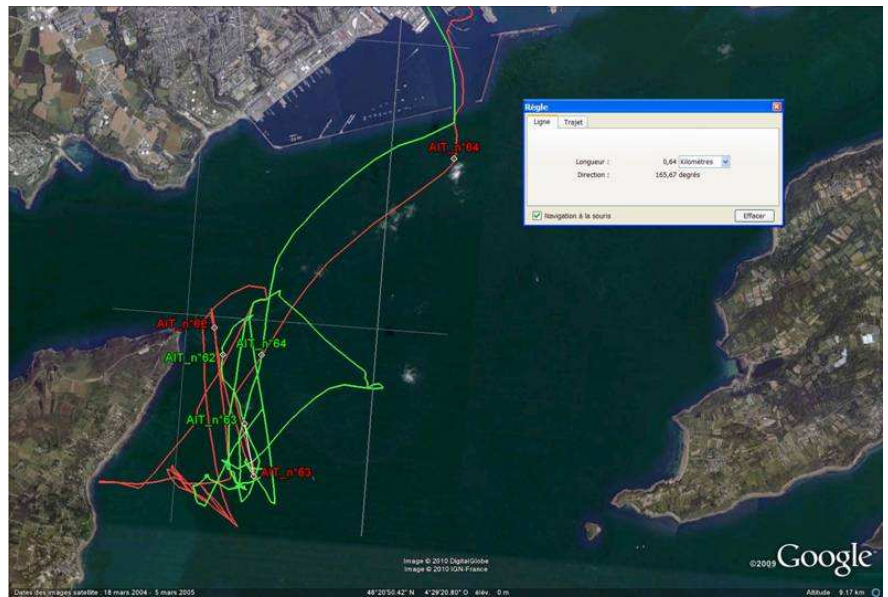

Fig. 4. Brest bay sea trial: record position for the sequence AIT63

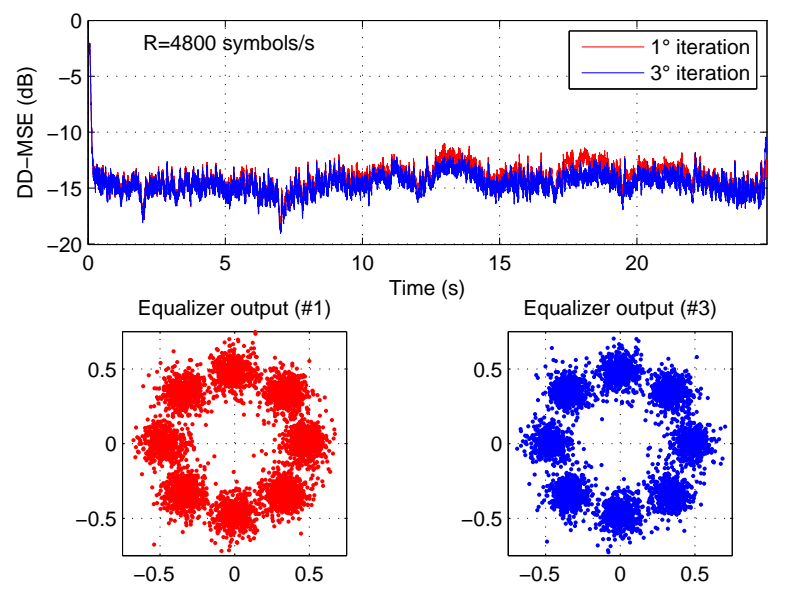

Fig. 5. Sequence AIT44: DD-MSE at the output of the multiple input equalizer, 8PSK, 4800 symbols/s, channel bit rate: $14400 \mathrm{bps}, f_{c}=17.5$ $\mathrm{kHz}$, distance $=1530 \mathrm{~m}, \mathrm{v}=0.26 \mathrm{~m} / \mathrm{s}$

To build a burst of symbols, a rate $R_{c}=1 / 2$ convolutional code with constraint length 5 was fed by a block of binary data $\alpha_{n}$. An interleaver $\Pi$ shuffled 22500 coded data $c_{n, i}$ and provided interleaved coded data $c_{k, i}$. Each set of $m=\log _{2}(M)$ interleaved coded data $c_{k, i}$ was associated with M-ary complex symbol $d_{k}$ by a Gray mapping. A transmitted burst resulted on the concatenation of one preamble of 1 second followed by a repetition of $m=\log _{2}(M)$ blocks of $22500 / \log _{2}(M)$ symbols separated by a pilot sequence of 256 symbols. The burst duration was 25 seconds. The ratio between the user bit rate and the channel bit rate was approximately 0.45 .

In order to emphasize on the improvements with the proposed turbo equalizer, we show the behavior of the receiver in terms of decision-directed minimum mean square error (DD-MSE) at the equalizer output versus the iteration number over a duration of 25 seconds. 

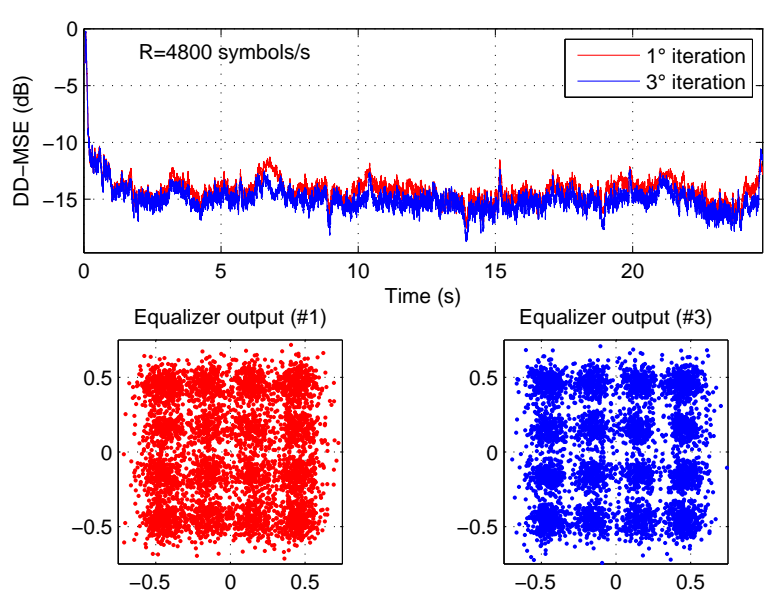

Fig. 6. Sequence AIT44: DD-MSE at the output of the multiple input equalizer, 16QAM, 4800 symbols/s, channel bit rate: $19200 \mathrm{bps}, f_{c}=17.5$ $\mathrm{kHz}$, distance $=1530 \mathrm{~m}, \mathrm{v}=0.26 \mathrm{~m} / \mathrm{s}$

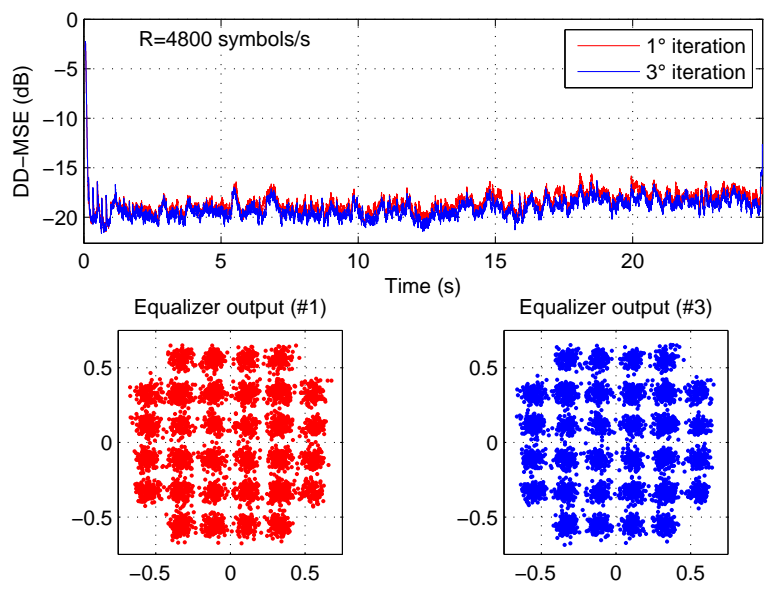

Fig. 7. Sequence AIT63: DD-MSE at the output of the multiple input equalizer, 32QAM, 4800 symbols/s, channel bit rate: $24000 \mathrm{bps}, f_{c}=17.5$ $\mathrm{kHz}$, distance $=640 \mathrm{~m}, \mathrm{v}=2 \mathrm{~m} / \mathrm{s}$

The DD-MSE is estimate adaptively by $D D M S E_{k+1}=$ $\lambda D D M S E_{k}+(1-\lambda)\left|z_{k}-\hat{d}_{k-\Delta}\right|^{2}$ where $\lambda=0.99$ is a forgotting factor. As seen in Figures 5, 6 and 7, the performance between the first iteration and third iteration is improved at the equalizer output. The constellations are plotted for 1 second duration (4800 symbols) from the 10th to the 11th second.

In Tables III and IV, we give the bit error rate (BER) at the equalizer and decoder outputs versus the iteration. BER was computed on a duration of 25 seconds. The BER at the equalizer output is slightly improved between the first and the third iteration of the turbo equalizer. Since, the SNR is lower for the sequence AIT44, the equalizer fails for the 32QAM
TABLE III

SEQUENCE AIT44: PERFORMANCE RESULTS IN BER

\begin{tabular}{|l|c|c|c|}
\hline Sequence AIT44 & 8 -PSK & $16-\mathrm{QAM}$ & $32-\mathrm{QAM}$ \\
\hline BER equalizer output (1st iteration) & $6.7 \mathrm{e}-3$ & $1.9 \mathrm{e}-2$ & 0.5 \\
\hline BER decoder output (1st iteration) & $1.8 \mathrm{e}-5$ & $3.1 \mathrm{e}-4$ & 0.5 \\
\hline BER equalizer output (3rd iteration) & $5.6 \mathrm{e}-3$ & $1.1 \mathrm{e}-2$ & 0.5 \\
\hline BER decoder output (3rd iteration) & 0 & $3.1 \mathrm{e}-4$ & 0.5 \\
\hline
\end{tabular}

TABLE IV

SEQUENCE AIT63: PERFORMANCE RESULTS IN BER

\begin{tabular}{|l|c|c|c|}
\hline Sequence AIT63 & 8-PSK & 16-QAM & 32-QAM \\
\hline BER equalizer output (1st iteration) & $4.1 \mathrm{e}-4$ & $1.1 \mathrm{e}-3$ & $9.1 \mathrm{e}-3$ \\
\hline BER decoder output (1st iteration) & 0 & 0 & $1 \mathrm{e}-4$ \\
\hline BER equalizer output (3rd iteration) & $4.1 \mathrm{e}-4$ & $1.1 \mathrm{e}-3$ & $7 \mathrm{e}-3$ \\
\hline BER decoder output (3rd iteration) & 0 & 0 & $2.1 \mathrm{e}-5$ \\
\hline
\end{tabular}

modulation and the bit error rate is 0.5 .

For these two sequences (AIT44 and AIT63), the performance gain between the first and the third iteration of the turbo equalizer is weak because the intersymbol interference on the transmission is low. In the Figure 8 is depicted the channel state information (CSI) after Doppler shift compensation. Because it is well-known that the turbo equalization is essentially efficient when the frequency selectivity is high, the performance results obtained for these real sea experiments are in accordance.

\section{Conclusions}

Robust single carrier transmissions of high order modulations have been performed in real sea environment with user data rate up to $10 \mathrm{kbps}$. Unfortunately, for this experimental campaign, only two sequences with high order modulation were recorded. From these two sequences, the turbo equalizer
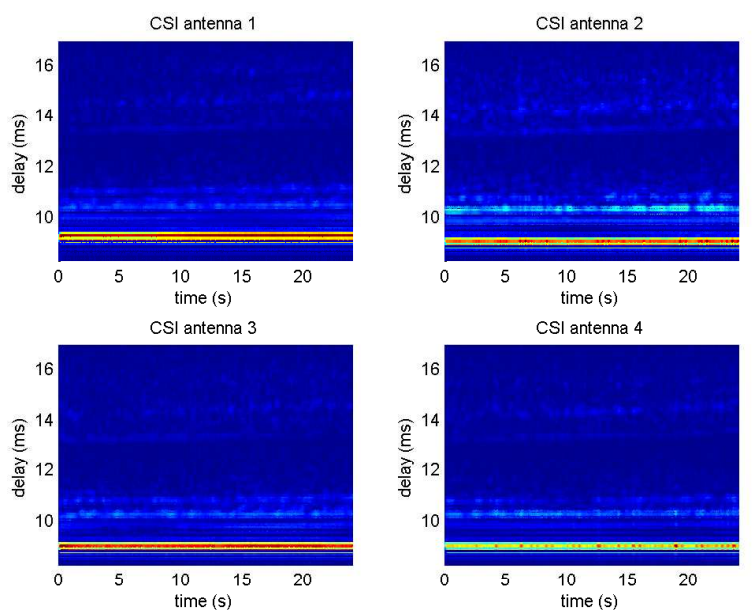

Fig. 8. Sequence AIT63: channel state information, 4800 symbols/s, $f_{c}=$ $17.5 \mathrm{kHz}$, distance $=640 \mathrm{~m}, \mathrm{v}=2 \mathrm{~m} / \mathrm{s}$ 
improves the equalization performance but the gain in term of bit error rate at the decoder output is weak. The advantage of the iterative equalization process with regards to the additional complexity is not emphasized. It is probable that if the channel is more frequency selective, the performance gain in term of bit error rate will be better. New real sea experiments will be carried in september 2010 with the objective to have additional recorded signals.

\section{REFERENCES}

[1] J. Trubuil, G. Lapierre, and J. Labat, "Real time transmission of images and data through underwater acoustic channel: the trident system," in Proc. of IGARSS'04, Anchorage, USA, 2004.

[2] A. Glavieux, C. Laot, and J. Labat, "Turbo equalization over a frequency selective channel," Proc. of Int. Symp. Turbo Codes, pp.96-102, Sept. 1997.

[3] M. Tuchler, R. Koetter, and A. Singer, "Turbo equalization: principle and new results," IEEE Trans. Comm., vol. 50,no. 5, pp. 754-767, May 2002.

[4] X. Wang and H. Poor, "Iterative (turbo) soft interference cancellation and decoding for coded CDMA," IEEE Trans. Commun., vol. 47, no. 7, pp.1046-1061, July 1999.

[5] E. Sangfelt, T. Oberg, B. Nilsson, and M. Lundberg Nordenvaad, "Underwater acoustic communication experiments in the baltic sea," in Proc. of Undersea Defence Technology UDT 2008, 2008.

[6] J. Choi, R. Drost, A. Singer, and J. Preisig, "Iterative multi-channel equalization and decoding for high frequency underwater acoustic communications," in Proc. of IEEE Sensor Array and Multichannel signal processing workshop, SAM 2008, 2008.

[7] C. Polprasert and J. Ritcey, "Performance of the bit-interleaved frequency domain turbo equalization over experimental underwater acoustic channels," in Proc. of Asilomar conference on signals, Systems and computers, Pacific grove, CA, 2008.

[8] R. Otnes and T. Eggen, "Underwater acoustic communications: Longterm test of turbo equalization in shallow water," IEEE Journal of Oceanic engineering, vol. 33, pp. 321-334, July 2008.

[9] C. Laot and P. Coince, "Experimental results on adaptive mmse turbo equalization in shallow underwater acoustic communication," Proc. of OCEANS'10, Sydney, Australia, May 2010.

[10] H. Meyr, M. Moeneclaey, and S. Fechtel, Digital Communication Receivers: Synchronization, Channel Estimation, and Signal Processing. New York: Wiley, 1998.

[11] M. Stovanovic, "Guest editorial: Underwater wireless communications," IEEE Communications Magazine, vol. 47, p. 78, Jan. 2009.

[12] J. Heidemann, U. Mitra, J. Preisig, M. Stovanovic, and M. Zorzi, "Guest editorial: Underwater wireless communications networks," IEEE Journal on Selected Areas in communications, vol. 26, pp. 1617-1616, Dec. 2008.

[13] B. Sharif, J. Neasham, O. Hinton, and A. E. Adams, "A computationaly efficient doppler compensation system for underwater acoustic communications," IEEE J. Oceanic Eng., vol. OE-25, pp. 52-61, Jan. 2000.

[14] L. Freitag, M. Johnson, and D. Frye, "High-rate acoustic communications for ocean observatories-performance testing over a $3000 \mathrm{~m}$ vertical path," Proc. of OCEANS'O0, pp. 1443-1448, Sept. 2000.

[15] B. Li, S. Zhou, L. Freitag, and P. Willet "Multicarrier communication over underwater acoustic channels with nonuniform doppler shifts," IEEE Journal of Oceanic Engineering, Vol. OE-33, pp. 198-209, Apr. 2008.

[16] J. Tao, Y.R. Zheng, C. Xiao, T.C. Yang and W.B. Yang "Channel Estimation, Equalization and Phase Correction for Single Carrier Underwater Acoustic Communications," Proc. of OCEANS'08, Kobe, Apr. 2008.

[17] Q. Cai, A. Wilzeck, and T. Kaiser, "A compound method for initial frequency acquisition in wcdma systems," in IEE DSP Enabled Radio Conference, Southampton, England, 2005.

[18] G. Eynard and C. Laot, "Blind doppler compensation scheme for single carrier digital underwater communications," in Proc. of OCEANS'08, Quebec, Canada, 2008.

[19] F. Gardner, Demodulator Reference Recovery Techniques Suited for Digital Implementation, ser. ESTEC Contract No. 6847/86/NL/DG. European Space Agency, 1988.
[20] C. Laot, R. Le Bidan, and D. Leroux, "Low complexity linear turbo equalization: A possible solution for EDGE," IEEE Trans. Wireless. Comm., vol. 4, no. 3, pp 965-974, May 2005. 